\title{
The Challenge of Learning for Networked SMEs to Increase Competitiveness in Virtual Enterprises
}

\author{
Heiko Duin ${ }^{1}$, Manuel Oliveira ${ }^{2}$, Sobah Abbas Petersen ${ }^{2}$, and Klaus-Dieter Thoben ${ }^{1}$ \\ ${ }^{1}$ BIBA - Bremer Institut für Produktion und Logistik GmbH, \\ Hochschulring 20, D-28359 Bremen, Germany \\ $\{d u$, tho\} @biba.uni-bremen.de \\ ${ }^{2}$ SINTEF Technology and Society, \\ S.P. Andersens vei 5, 7465 Trondheim, Norway \\ \{manuel.oliveira, sobah. petersen\} @sintef.no
}

\begin{abstract}
Collaborative Networked Organisations (CNO) face the same if not even harder problems when trying to transform towards a learning organisation. Small and medium sized organisations - which still represent the motor for job creation in Europe - often respond to market turbulence by engaging in nonhierarchical CNOs such as Virtual Enterprises. A proven method to support learning in distributed set-ups is Serious Gaming, but the question remains whether the application of such tools is equally effective in SMEs compared with monolithic large organisations. In SMEs and networks of SMEs learning needs to move towards contextualised learning and a serious gaming based transformative environments such as TARGET can be used to achieve learning goals and competence development in distributed set-ups. This paper introduces such a transformative environment and highlights the background, its usage, benefits and limitations.
\end{abstract}

Keywords: Learning, Collaborative Networked Organisations, SMEs, Serious Gaming, Transformative Environments.

\section{Introduction}

In the global market, with the emergent economies, European enterprises have serious difficulties in surviving, let alone excelling, unless they are capable to leverage successfully their capacity to learn and to innovate. Especially, within the knowledge economy, a key survival factor for organizations facing an uncertain turbulent business environment is their ability to change, adapt and evolve, exploring new opportunities as they emerge and drive the organization towards the realization of its full potential. Often, enterprises - and more specific small and medium sized enterprises (SMEs) address emerging business opportunities in Collaborative Networked Organisations (CNOs) such as Virtual Organisation Breeding Environments (VBEs) and Virtual Organisations (VOs) (Camarinha-Matos et al., 2009).

However, the willingness to cooperate and the installation of management procedures for the networked organisation alone is not a key for being successful. 
According to Senge, the only way for organizations to maintain market competiveness is when "people continually expand their capacity to create the results they truly desire, where new and expansive patterns of thinking are nurtured, where collective aspiration is set free, and where people are continually learning to see the whole together" (Senge, 1990).

This implied agility and holistic approach requires collaborative design, innovation and learning at all levels of the networked organization, but also converting learning into collaborative action. While being anchored in individual learning, organizational learning means much more than just the sum of the parts of individual learning. Of course, learning as the cognitive process takes place at the individual level, but there is clearly an organizational phenomenon connected with it. Individual learning can be considered organizational when it is done to achieve organization purposes or the learning outcome is shared or among members of the organization and/or network or learning outcomes are somehow embedded in the organizations' systems, structures, and culture (Snyder \& Cummings, 1998).

Organisational Learning in a Network of SMES faces different challenges coming from being a SME and from being engaged in a Collaborative Network. SMEs often don't have a formalised competence development process. Daily business is in the foreground and learning takes place on-the-job or when there is some time left (which is never planned, but occur occasional). In almost all cases SMEs do not have an explicit budget for learning and competence development. Employees are highly specialised and enterprise culture (including language) can be very specific. If these SMEs are involved in a Collaborative Network additional challenges show up: while all the individuals involved in a Virtual Organisation are specialists in their specific area they may have just a little knowledge and understanding of other areas or the general objectives of their involvement in collaboration. In such a context, collaboration is an essential dimension of the learning process. Working and learning should be interleaved processes in a networked organization. The reduction of cost of learning and to potentially increase work quality makes it necessary to have a smooth transition between work and learning - on individual, organizational and network level.

Besides collaborative learning a participative approach to learning, called also "learner-centred", "constructivism", or "problem-based" is necessary. Such an approach implies active exploration, construction, and active learning rather than the passive attendance at lectures or the reading of textbooks (Cristea \& Florea, 1999).

Serious Gaming has proven to support learners in acquiring new and complex knowledge and is ideally suited to support problem based learning by creating engaging experiences around a contextual problem where users must apply competences to solve specific challenges (e.g. Duin et al., 2012). The advent of serious games has given rise to the possibility of enhancing the learning (Freitas, 2006) with an increasing number of advocates promoting the use of serious games as a delivery platform (Aldrich, 2005) for education and competence development. This effort has been hampered by the perceived lack of concrete evidence concerning the effectiveness of learning and the fragmentation of the research community raises difficult challenges (Hauge et al, 2010). However, gradual well-designed studies 
begin to emerge demonstrating that learning does take place, albeit it remains debatable if the results can be expounded to cover other learning domains, or even applications, since context plays a key role in how well a person performs a particular activity.

For cases where it is recognized that learning outcomes have been achieved, some argue the result was not derived from intentional pedagogical design (Gee, 2003). A special difficulty with the development of entertainment games, the design of serious games is very much a craft, which in the case of serious games is compounded by the challenge of making an engaging game that is "fun", but at the same time, it needs to be "serious" to support situated learning contexts where learners can acquire knowledge, abilities and skills.

The objective of this paper is create awareness for transformative environments as personalized and collaborative learning systems to support competence development of team members which are typically geographically distributed when considering collaborative networked organisations. Such a transformative environment is developed by the EU project TARGET and trialled with three different end user scenarios concerning complex project management, social composition and management of (distributed) teams and the ability to perform a Lifecycle Assessment (LCA) for the whole life cycle of a consumer product. All these scenarios contain the potential to be executed collaboratively in a networked context.

\section{Related Research}

Early research in learning in Virtual Organisations focussed on the creation and management of tacit and explicit knowledge assets (Steil, Barcia, and Pacheco, 1999). With moving focus from knowledge management related aspects towards the management of individual and organisational competences, CNO related research focussed on competence management like recent publications from the Pro-VE community show (e.g. Klamma \& Petrushyna, 2010, Fazel-Zarandi \& Fox, 2010, and Moreira et al, 2010).

Klamma and Petrushyna (2010) used a pattern-oriented approach to manage and develop competences in networked organisations. Pattern recognition is done by applying techniques from Social Network Analysis (SNA). Communities of Practice in an academic environment were the test cases from which a new modelling approach called $i^{*}$ modelling technique has been developed supporting competence development and management tasks.

Fazel-Zarandi and Fox (2010) address the problem of competence development through the view of Human Resource Management (HRM). A formal ontology has been developed which allows formal reasoning about competence development problems. Special focus has been set to the problems of identifying the set of skills of individuals, conducting competence gap analysis and testing whether a selected individual matches a given set of competence requirements.

Moreira et al (2010) developed a conceptual framework for the management of organisational competences to contribute to the evolution of business model of an 
enterprise. Results of their study involving automotive and furniture industry showed that the framework has high potential for ex-ante strategy formulation (strategic planning) and ex-post strategy explanation. "Soft" concepts such as leadership were identified as important with further research needs.

A high-level architecture for personalised learning in CNOs has been presented by Afsarmanesh and Tanha (2010). The authors point out that in collaborative networks the involved individuals are not typical learners compared to academic environments, and therefore it is even more important to make the learning time/cost effective. The personalized learning approach suggested in their paper aims to carefully apply both the learner's and the environment's characteristics into the customization of the most suitable reduced list of Learning Objects, as well as the near optimal learning path.

The efforts presented by these researches mainly focus on the identification of competence gaps on either individual or (inter-)organisational level and how to manage the closing of these gaps. Specific SME related challenges have not been addressed and the acquisition of "soft" skills through a Serious Gaming based approach is not considered in above mentioned research.

There is a need to capture the situation or the work context and to support learning in the workplace or situated learning (Lave \& Wenger 1991). As Fig. 1 says, there is a need to capture context and transform our perceptions of the environment. Working contexts, or work-spaces, can be developed as a set of models which can enable common and role-specific views to be derived and shared, and hence our perceptions will change, and we will learn as work and collaboration progresses (Lillehagen, Krogstie, 2008).

SME's often do not have the time or the resources to invest in learning. However, there sustainability is dependent on their ability to adapt to the changing face of business and the needs of their customers in a timely manner. SMEs can benefit enormously from peer learning by having access to the experiences of others either from other SMEs or larger organisations, The ideas of Communities of Interest of Communities of Practice (Wenger, 2002) can be a powerful tools in designing learning support for SMEs.

\section{Innovative Transformative Environments}

In TARGET, the learning process draws heavily from Problem Based Learning (PBL) and Action Learning (AL), resulting in the use of digital interactive stories in a serious game environment that provide situated rich contexts where a learner is required to apply and develop competences to achieve successful outcomes.

The situated contexts captured by Stories represent a scoped business environment where multiple characters are defined with specific roles and responsibilities. Since the paradigm of emergent storytelling is adopted, some of the characters are strategically controlled by Non-Player Characters (NPC) to ensure the Story unfolds with the aim of developing the associated competences. The aim is for the Project Manager to improve his/her competences, such as conflict management, negotiation and communication, 
through the experiences in the game. So for example, with conflict management, the NPC will control the anti-protagonist to oppose the learner's (e.g. the Project Manager's) goals. However, taking aside these strategic characters assumed by NPCs, the learner may choose which one of the remainder characters to assume in the Story. In the cases where there is more than a single character available for the learner to choose, then it is possible to have multiple learners engaged in the same Story. The fact that a few learners may simultaneously engage with the same Story does not change that learning continues to be individual irrespective of the possibility of learners exhibiting behaviours out-of-character, communicating with one another.

In addition to the virtual business environment provided in TARGET, it also includes an arena for the learners and other users to socialise virtually, called the Lounge". Learners are able to meet other users in the Lounge and access "experiences" (the capture of a learner's experience through the game) of other learners. Capabilities supported in the Lounge support peer learning as well as the social aspects of learning, through ideas such as Communities of Practice and Communities of Interests.

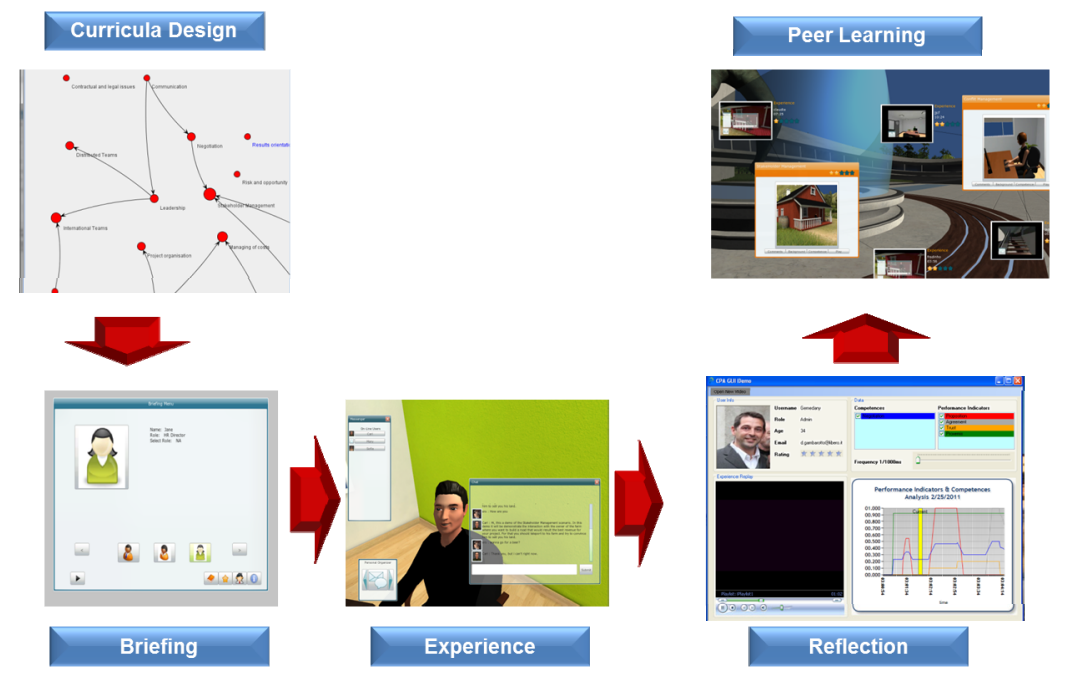

Fig. 1. Overview of the TARGET Learning Process

The instantiation of the TARGET Learning Process, which is supported by a componentized platform, is illustrated in the diagram of Fig. 1. Each of the stages in that diagram can be characterized as follows:

- Curricula Design. The TARGET Learning Process begins with the learner deciding on what competences to develop. This is done in one of two ways, either goal-oriented or self-directed learning. In the case of goal-oriented learning, the learner defines their current competence profile and their desired learning outcome in the form of outlining their target competence profile. The result of profiling leads to the creation of a learning plan based on custom stories tailored to the particular needs of the learner. Each story captures a 
business context, which may also involve defined characters with particular roles. The process of creating the learning plan is governed and shaped by a learning strategy that is chosen by the learner. In the case of self-directed learning, the learner builds their learning plan from the experiences of others within the community and these are stored in the knowledge ecosystem.

- Briefing. The learner is provided a background to a Story, which gives insight into the context, including the various characters available and their role in the Story. Some of the characters are available to the learner to be played by them, but in many cases the characters are only manipulated by NPCs.

- Experience. Whilst engaged with the Story, the system provides an environment where the learner engages with other characters (either controlled by another learner or a NPC) and the environment, enacting their decisions. These decisions will have an impact which will affect and change the situated context of the Story. By monitoring the actions of the learner and taking into account the desired learning outcomes, the TARGET platform makes changes to the Story if necessary. As examples, these changes may be modifying the personality of a NPC to be more confrontational or delaying tasks within a project.

- Reflection. The learner is presented with the assessment of their competence during the experience in the form of a timeline manner. The ability of looking back on their decisions by reviewing how the story unfolded whilst crossreferencing the assessment of their competence at each point in time, allows the learner to evaluate their performance leading to reflection.

- Peer Learning. The TARGET learning process supports the learner in externalizing the tacit knowledge acquired after their experience of a Story, thereby contributing to the creation of knowledge assets that are uploaded to the Knowledge Ecosystem. Once uploaded, the learning community plays an important role in the process with the support of recognized mentors as facilitators and in discussion with other learners. The social aspects address the need of an ability to deal with flux and instability, and to thrive in situations of flux.

Each of the five phases of the TARGET learning process is supported by a set of well-defined services embodied into components that are event driven, thus loosely decoupled from one another with some sharing functional dependencies. This means that the TARGET platform need not be entirely deployed as an integrated solution, but only subsets of the supported functionality. However, one needs to ensure that those components sharing functional dependencies are deployed together otherwise they may be operational at run-time but not work as required.

One of challenges of this approach is to enrich the set of stories or game scenarios that are available for the learners in the TARGET environment. Similarly, to support peer learning, learners or other users need to annotate the experiences that are captured in the system. This is perhaps one of the important aspects of learning support for SMEs where they are able to capitalise on the experiences of their peers, thus saving time and effort that is used in learning. 


\section{Conclusions}

This paper addresses the challenges of learning and competence development in CNOs, in particular, SMEs. Initiatives to support formalised learning for employees in an organisation have been mostly conducted by larger organisations, while SMEs often do not have the means or the time to engage in such activities. The objective of this paper is to create awareness for transformative environments as personalized learning systems to support competence development of team members which are typically geographically distributed. Serious gaming has proven to be an effective means of supporting situated and contextualised learning within a work environment. We believe that serious gaming will meet the learning needs of SMEs, supporting experiences based learning that is focused to the situation at hand, thus reducing the time and effort that is required to rapidly gain competences in relevant areas. In SMEs and networks of SMEs, learning needs to move towards contextualised learning and a serious gaming based transformative environments such as TARGET can be used to achieve learning goals and competence development in distributed set-ups. This paper introduces such a transformative environment and highlights the background, its usage, benefits and limitations.

Acknowledgements. This work has been partly funded by the European Commission through ICT Project TARGET: Transformative, Adaptive, Responsive and enGaging Environment (No. ICT-231717). The authors wish to acknowledge the Commission for their support. We also wish to acknowledge our gratitude to all TARGET project partners for their contribution.

\section{References}

1. Afsarmanesh, H., Tanha, J.: A High Level Architecture for Personalized Learning in Collaborative Networks. In: Camarinha-Matos, L.M., Boucher, X., Afsarmanesh, H. (eds.) PRO-VE 2010. IFIP AICT, vol. 336, pp. 601-608. Springer, Heidelberg (2010)

2. Aldrich, C.: Learning by doing: a comprehensive guide to simulations, computer games, and pedagogy in e-learning and other educational experiences. Pfeiffer (2005)

3. Baldwin, J.: Social Institutions: the School, the State, the Church. In: Badger (ed.) The Individual and Society or Psychology and Sociology, Boston, pp. 118-144 (1911)

4. Camarinha-Matos, L.M., Afsarmanesh, H., Galeano, N., Molina, A.: Collaborative Networked Organizations - Concepts and Practice in Manufacturing Enterprises. Computers and Industrial Engineering 57, 46-60 (2009)

5. Cristea, V., Florea, A.: Concurrent learning in virtual organisations. In: Proceedings of ICE 1999, The 5th International Conference on Concurrent Enterprising, Hague, The Netherlands, March 15-17, pp. 385-392 (1999)

6. Duin, H., Oliveira, M., Thoben, K.-D.: A Methodology for Developing Serious Gaming Stories for Sustainable Manufacturing. In: 18th International ICE-Conference on Engineering, Technology and Innovation (accepted paper 2012) 
7. Fazel-Zarandi, M., Fox, M.S.: Reasoning about Skills and Competencies. In: CamarinhaMatos, L.M., Boucher, X., Afsarmanesh, H. (eds.) PRO-VE 2010. IFIP AICT, vol. 336, pp. 372-379. Springer, Heidelberg (2010)

8. Flanagan, M., Taylor, P., Meyer, J.: Compounded Thresholds in Electrical Engineering. In: Land, R., Meyer, J.H.F., Baillie, C. (eds.) Threshold Concepts and Transformational Learning. Sense Publishers, Rotterdam (2010)

9. de Freitas, S.: Using games and simulations for supporting learning, Learning. Media and Technology 31, 343-358 (2006)

10. Gee, J.: What video games have to teach us about learning and literacy. Palgrave Macmillan, New York (2003)

11. Hauge, J.B., Riedel, J., Fradinho, M., Westra, W.: Addressing Research Fragmentation in Serious Gaming for Manufacturing. In: 13th International Workshop of the Special Interest Group on Experimental Interactive Learning (2010)

12. Klamma, R., Petrushyna, Z.: Pattern-Based Competence Management: On the Gap between Intentions and Reality. In: Camarinha-Matos, L.M., Boucher, X., Afsarmanesh, H. (eds.) PRO-VE 2010. IFIP AICT, vol. 336, pp. 364-371. Springer, Heidelberg (2010)

13. Lave, J., Wenger, E.: Situated Learning: Legitimate Peripheral Participation. University Press, Cambridge (1991)

14. Lillehagen, Krogstie: Active Knowledge Modelling of Enterprises. Springer, Heidelberg (2008)

15. Moreira, M.P., D'Amours, S., Beauregard, R., Azouzi, R.: The Role of Organizational Competences in the Evolution of Business Models. In: Camarinha-Matos, L.M., Boucher, X., Afsarmanesh, H. (eds.) PRO-VE 2010. IFIP AICT, vol. 336, pp. 396-403. Springer, Heidelberg (2010)

16. Senge, P.: The Fifth Discipline. The Art and Practice of the Learning Organization, New York (1990)

17. Snyder, W., Cummings, T.: Organizational Learning Disorders: Conceptual Model and Intervention Hypotheses. Human Relations 51(7), 873-895 (1998)

18. Steil, A.V., Barcia, R.M., Pacheco, C.S.: An approach to learning in virtual organizations. Electronic Journal of Organizational Virtualness (eJOV) 1(1), 69-88 (1999)

19. Wenger, E., McDermott, R., et al.: Cultivating Communities of Practice: A Guide to Managing Knowledge. Harvard Business School Press (2002) 